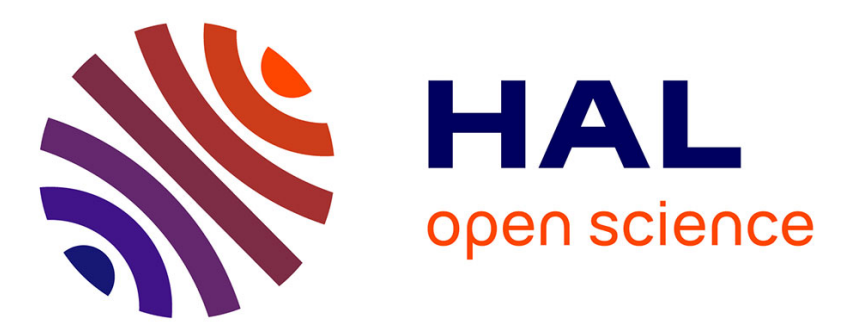

\title{
Experimental validation of the diffusion function model for accuracy-enhanced thermoreflectometry
}

Benjamin Javaudin, Rémi Gilblas, Thierry Sentenac, Yannick Le Maoult

\section{To cite this version:}

Benjamin Javaudin, Rémi Gilblas, Thierry Sentenac, Yannick Le Maoult. Experimental validation of the diffusion function model for accuracy-enhanced thermoreflectometry. Quantitative InfraRed Thermography Journal, 2021, 18 (1), pp.18-33. 10.1080/17686733.2019.1665297 . hal-02384426

\section{HAL Id: hal-02384426 \\ https://imt-mines-albi.hal.science/hal-02384426}

Submitted on 12 Feb 2020

HAL is a multi-disciplinary open access archive for the deposit and dissemination of scientific research documents, whether they are published or not. The documents may come from teaching and research institutions in France or abroad, or from public or private research centers.
L'archive ouverte pluridisciplinaire HAL, est destinée au dépôt et à la diffusion de documents scientifiques de niveau recherche, publiés ou non, émanant des établissements d'enseignement et de recherche français ou étrangers, des laboratoires publics ou privés. 


\title{
Experimental validation of the diffusion function model for accuracy-enhanced thermoreflectometry
}

\author{
Benjamin Javaudin, Rémi Gilblas, Thierry Sentenac and Yannick Le Maoult \\ Institut Clément Ader (ICA), Université de Toulouse, CNRS, IMT Mines Albi, UPS, INSA, ISAE-SUPAERO, \\ Toulouse, France
}

\begin{abstract}
In the field of thermography, the main problem in measuring true temperature lies in determining the emissivity. Thermoreflectometry overcomes this problem by an in-situ emissivity determination method based on bidirectional reflectivity measurement. This method introduces a diffusion function model which links the bidirectional reflectivity measured to the emissivity. The true temperature and the model parameters are then found with the solution of a system of radiometric equations. The first contribution of this article is the modelling of the diffusion function using physical models of the Bidirectional Reflectivity Distribution Function (BRDF). The suggested model depends on two parameters: the roughness and the geometrical setting of the measurement. For a given configuration, the identification of the roughness parameter provides a knowledge of the evolution of the surface state according to the operating conditions of the material (deformation, damage, etc.). The second contribution is the validation of the roughness parameter identified by the thermoreflectometry method. Validation is done on a reference sample in platinum with a measured roughness. Another validation is performed on the BRDF model by measurements on a Fourier transform spectrometer. It validates the shape of the model and gives a second validation of the roughness parameter by using curve-fitting identification.
\end{abstract}

\author{
KEYWORDS \\ BRDF; modelling; \\ thermoreflectometry; \\ emissivity; active \\ thermography
}

\section{Introduction}

Temperature is a key parameter in many industrial processes and its measurement by non-contact techniques like thermography offers many advantages compared to contact techniques. This method has the capability to provide radiance temperature measurement but it requires the knowledge of the surface emissivity to compute the true temperature. The emissivity can be taken into account by following two approaches: passive and active thermography.

For monochromatic thermography, the impact of the lack of emissivity knowledge can be minimised by operating the camera in the ultraviolet band [1]. Polychromatic thermography [2] models the behaviour of the emissivity for several wavelengths and solves a system composed of spectral radiometric equations. To do so, the temperature and the 
parameter(s) of the emissivity model are estimated. This method assumes that the variation of the emissivity is consistent with the modelling throughout the duration of the experiment. However, this assumption can be quickly obsolete because of the evolution of surface-state phenomena like oxidation, ablation or phase transition.

Active thermography overcomes the emissivity variation problem by including an "insitu" emissivity determination. For instance, thermoreflectometry $[3,4]$ suggests an indirect measurement of emissivity based on bidirectional reflectivity measurement. For an opaque material, directional emissivity is linked to directional-hemispherical reflectivity through Kirchhoff's law. Thermoreflectometry then introduces a relationship between these two quantities through a diffusion function. It is a model whose parameters are estimated by the resolution of a multi-wavelength system composed of radiometric equations. This resolution then gives the true temperature measurement and the diffusion function parameter. Earlier work assumed that the diffusion function was only a constant between two close wavelengths [3]. This assumption means that the reflectivities are homothetic at two wavelengths. This model gives an indication of the nature of the reflection (a low value is the property of a specular surface and, respectively, a high value of a diffuse surface) but it does not characterise the physical property of the surface, in terms of roughness, for example.

This article aims to model the diffusion function physically, with parameters depending on surface properties such as roughness. For opaque and strong absorbing material like metallic materials, the interaction between light and roughness is a surface-scattering phenomenon because of the very thin penetration depth of the radiation. Assuming that the roughness is much larger than the wavelength, analytical models in the context of the geometrical optics approximation (GOA) explain the surface-scattering phenomenon and are established from the Bidirectional Reflectivity Distribution Function (BRDF). These models, widely used in the realistic image synthesis community, describe the roughness statistically as facets whose slope distributions are modelled by a probability density function. Each facet then scatters the light as elementary specular reflections modelled by the Fresnel equations. Thanks to these models, and considering only the case of Gaussian isotropic surface roughness, a new diffusion function is suggested which depends on the roughness parameter and the geometrical setting of the experiment. The application of this function in the bi-chromatic thermoreflectometry method, where geometry is known, then provides an estimation at the same time of the true temperature and the roughness parameter.

Various tests were conducted for a first validation of the diffusion function model on a reference sample with a controlled roughness. The material chosen was the platinum because its optical properties are well known in the literature and it is chemically stable at high temperature. The first test was the roughness profile measurement, carried out with a confocal profilometer. It validated the slope distribution of the facets and it provided a reference roughness parameter which is the standard deviation of these slopes. The second test was a comparison between the BRDF model, used in the diffusion function, and the BRDF measured by a Fourier transform spectrometer. It validated the shape of the model with regard to the experimental BRDF by a curve-fitting method. The test also provides a second validation of the roughness parameter due to the parameter identification. The last test was an estimation of the roughness parameter by the bi-chromatic thermoreflectometry method using the diffusion function model on the heated platinum 
sample at several temperature. These third values were compared to others provided in other tests at room temperature. Uncertainty budgets are not presented in this paper which is focus on the modelling and the experimental validation loop presented on a reference sample.

The paper is organised as follows: section 2 is dedicated to the introduction of the diffusion function in the thermoreflectometry method. Section 3 presents the modelling approach and concludes by a simulation of the new diffusion function model. Section 4 is devoted to the experimental validations of the diffusion function model.

\section{Introducing of the diffusion function in thermoreflectometry}

Thermoreflectometry is an active method which combines two steps: thermography and reflectometry. The thermography step consists in measuring the radiance temperatures by inverting a radiometric model with coefficients estimated by a previous black-body calibration. The reflectometry step consists in measuring the bidirectional reflectivity, defined as follows:

$$
\rho_{\lambda}^{i, r}=\rho_{\lambda}\left(\theta^{i}, \theta^{r}, \phi\right)=\frac{d L_{\lambda}^{r}}{d E_{\lambda}^{i}}
$$

where $d L_{\lambda}^{r}$ is an elementary spectral radiance reflected and $d E_{\lambda}^{i}$ the elementary spectral irradiance. The unity of $\rho_{\lambda}^{i, r}$ is $\left[s r^{-1}\right]$.

Measuring this quantity is not enough to determine the emissivity $\left(\varepsilon_{\lambda}^{\vec{r}}\right)$ essential to determining the true temperature. Indeed, for an opaque material, Kirchhoff's law links the emissivity to the directional hemispherical reflectivity $\left(\rho_{\lambda}^{r, \cap}\right)$. Thermoreflectometry then introduces a diffusion function $\left(\eta_{\lambda}^{\vec{r}, \vec{i}}\right)$ in order to link the emissivity to the bidirectional reflectivity measured as follows:

$$
\varepsilon_{\lambda}^{r}=1-\rho_{\lambda}^{r, \cap}=1-\eta_{\lambda}^{r, i} \rho_{\lambda}^{i, r}
$$

where the exponent $r$ is the direction of the detector, $i$ the direction of the light source and $\cap$ represents the hemisphere up to the surface. The unit of $\eta_{\lambda}^{r, i}$ is $[s r]$.

This equation, associated with the radiometric equation at each wavelength measurement, forms the thermoreflectometric system, defined as follows:

$$
\left\{\begin{array}{l}
L_{0}\left(T_{R}^{r}\left(\lambda_{j}\right), \lambda_{j}\right)=L_{0}\left(T, \lambda_{j}\right) \times\left(1-\rho_{\lambda_{j}}^{i, r} \eta_{\lambda_{j}}^{r, i}\right) \\
j=j, 2, \ldots N
\end{array}\right.
$$

where $L_{0}\left(T_{R}^{r}\left(\lambda_{i}\right), \lambda_{i}\right)$ is the Planck function applied to the radiance temperature $T_{R}^{r}$ measured in direction $r$ and at wavelength $\lambda_{i}$. The diffusion function $\eta_{\lambda}^{r, i}$ and the true temperature $T$ are the unknowns of this system. $N$ is the number of wavelengths and equations.

The system of Equation (3) is $N+1$ unknowns (T and $\eta_{j}^{r, i}$ ) for $N$ equations. A spectral model of the diffusion function needs to be introduced in order to decrease the number of unknowns. For instance, in the bi-chromatic approach $[3,4]$, the system is solved assuming that the diffusion function is constant between two close wavelengths. 
Instead of using an empirical model, this work aims to model the diffusion function physically, so as to link it to a surface property, such as the roughness of the material. This involves the modelling of the interaction between light and roughness which is established in the Bidirectional Reflectivity Distribution Function (BRDF). Indeed, this function includes the directional reflectivity of a surface in the whole hemisphere. From Equation (2), the diffusion function is expressed with the BRDF as follows:

$$
\eta_{\lambda}^{r, i}=\frac{\rho_{\lambda}^{r, \cap}}{\rho_{\lambda}^{i, r}}=\frac{\int_{2 \pi} \rho_{\lambda}^{r, x} \cos \theta^{x} d \Omega^{x}}{\rho_{\lambda}^{i, r}}
$$

where $d \Omega^{x}$ is the solid angle in direction $x$ and forms an angle $\theta^{x}$ with the surface normal. The directions $i$ and $r$ are fixed quantities and $x$ takes all the values in the hemisphere $\Omega=2 \pi$.

Equation (4) shows that for a fixed surface and a fixed detector direction $(r)$, the diffusion function depends on the direction of the light source (i). Thus, the behaviour of the diffusion function depends on the choice of the geometrical settings $(r, i)$, in addition to the surface properties included in the BRDF. Modelling the diffusion function is thus an opportunity to find the sample properties and the best measurement settings.

In the next section, a BRDF model is presented and expressed in the directions $(i, r)$ and $(r, x)$ in order to deduce the diffusion function of Equation (4).

\section{Modelling the diffusion function from a BRDF model}

This section presents the modelling steps for the diffusion function. It starts with the presentation of BRDF models and the derivation of their associated functions. Then, this chosen model is used to build the diffusion function by means of Equation (4). Finally, a simulation of the diffusion function is presented for two geometrical settings and as a function of the roughness parameter.

\subsection{Choice of a BRDF model}

Modelling the reflective behaviour for scattering surfaces is relatively easy for specific surfaces. Fresnel's law well describes specular surfaces and Lambert's law can be used for perfectly scattering surfaces. For usual surfaces, the modelling is more complex and the interaction between the light and the surface roughness needs to be taken into account.

In order to describe the reflection process on rough surfaces, the geometrical optics approximation (GOA), also called ray optics, is used. This approach describes the light as a group of elementary rays which follow the Fresnel equations. This choice neglects the wavelike behaviour of the light and assumes that the size of the roughness is much larger than the wavelength. Quantitative criteria on roughness parameters can be found in reference [5], which compares wave theory computations and ray tracing (GOA) on the same generated Gaussian random surfaces.

To avoid computation of an explicit surface, the roughness can be taken into account in a statistical way. This approach is taken in Torrance and Sparrow's model [6] which combines geometric optics with a statistical description of the roughness in a micro-facet 
BRDF model. Each bidirectional reflectivity of the BRDF is then the consequence of the sum of elementary Fresnel reflections on micro-facets oriented in defined positions. Thus, the shape of the BRDF becomes linked to the statistical distribution function of the microfacet orientations. This approach takes only the first BRDF reflection into account and not the multi-reflexion, as is the case in the ray-tracing method [7]. This second-order effect is thus neglected for the next sections.

\subsection{BRDF modelling}

For the BRDF model in a given configuration $(i, r)$, a global coordinate system, shown in Figure 1 , is defined from the normal vector $(N)$ of the surface. The vector $\left(L^{i}\right)$ defines the direction of the incoming light and $\left(L^{r}\right)$ the outgoing direction. These two vectors define with $(N)$ the zenith angles noted $\left(\theta^{i}\right.$ and $\left.\theta^{r}\right)$. Finally, the azimuth angle $\phi$, between the projection of $L^{i}$ and $L^{r}$ on the plane of the surface (whose normal vector is $N$ ) generalises the definition of this global geometry in all the hemisphere. As a remark, the definition of $\phi$ allows a rotational symmetry of $\left(L^{i}, L^{r}\right)$ around the normal vector. In that case, the BRDF model also has this symmetry which is only true when the roughness does not depend on the sample orientation (isotropic roughness behaviour).

In addition to Figure 1, a local frame, represented in Figure 2, describes the reflection of the light on a micro-facet. $H$ is defined as the bisector vector of $\left(L^{i}, L^{r}\right)$. In this way, each micro-facet oriented by an angle $(a)$, is set to reflect the light from $L^{i}$ to $L^{r}$. The angle of this specular geometry is then noted $\beta$. Finally, for a given global geometry $\left(\theta^{i}, \theta^{r}, \phi\right)$, Torrance's model [6] is expressed in Equation (5).

$$
\rho_{\lambda}^{i, r}=\rho_{\lambda}\left(\theta^{i}, \theta^{r}, \phi\right)=\frac{F\left(\beta, n_{\lambda}, k_{\lambda}\right) D(a) G\left(\theta^{i}, \theta^{r}\right)}{4 \cos \theta^{i} \cos \theta^{r}}
$$

where the angles $(\alpha, \beta)$ are related to $\left(\theta^{i}, \theta^{r}, \phi\right)$ by the definition of $H=\frac{L^{i}+L^{r}}{\left\|L^{i}+L^{r}\right\|}$. Also, the exponents $(i, r)$ are switchable, which respects the reciprocity path theorem $\left(\rho_{\lambda}^{i, r}=\rho_{\lambda}^{r, i}\right)$.

The geometric attenuation factor function, $G\left(\theta^{i}, \theta^{r}\right)$, takes into account the shadowing and masking effects induced between the micro-facets. Shadowing arises when a microfacet obstructs the path of the incoming light and masking occurs when the outgoing path

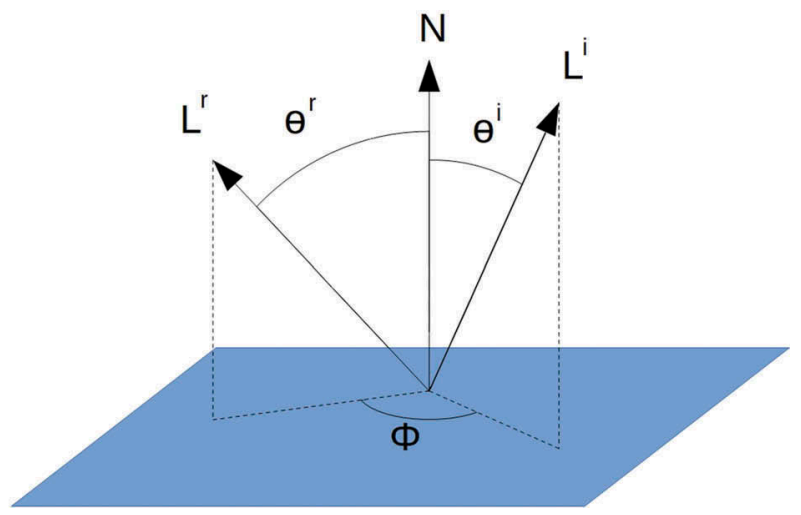

Figure 1. Global coordinate system with the units vector $L^{i}, L^{r}, N$ and the angles $\theta^{i}, \theta^{r}, \varphi$. 


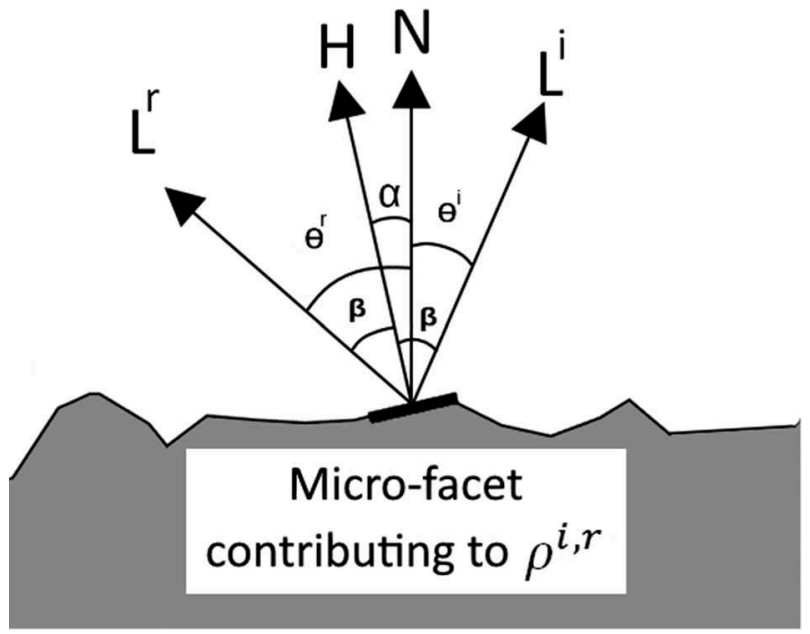

Figure 2. Local coordinate system on a micro-facet with the local normal vector $H, L^{i}$ and $L^{r}$ in the plane of the figure.

is obstructed. However, these two geometrical effects are strong only for grazing angles and do not depend on the wavelength. They should also be completed by a model of multireflection which is not considered in this study. Thus, the function $G$ will be neglected in this first version of the BRDF model to simplify the model.

The Fresnel function, $F\left(\beta, n_{\lambda}, k_{\lambda}\right)$, gives the specular reflectance of the micro-facet oriented by an angle $a$. The local incident and reflection angle is then $\beta$. This function includes the spectral dependence of the BRDF with the complex index of refraction $\left(n_{\lambda}, k_{\lambda}\right)$ associated with the material. In Equation (5), assuming that the angle $\beta$ stays close to zero, the Fresnel function, $F\left(\beta, n_{\lambda}, k_{\lambda}\right)$, is approximated by the normal Fresnel factor $f_{\lambda}^{0}$ as follows:

$$
F\left(\beta, n_{\lambda}, k_{\lambda}\right) \approx f_{\lambda}^{0}=\frac{\left(n_{\lambda}-1\right)^{2}+k_{\lambda}^{2}}{\left(n_{\lambda}+1\right)^{2}+k_{\lambda}^{2}}
$$

where $n_{\lambda}$ is the refractive index and $k_{\lambda}$ the imaginary part of the complex refractive index. The benefit of this approximation is to replace the optical index by only one variable $f_{\lambda}^{0}$.

The distribution function, $D(a)$, describes the amount of energy reflected by all the micro-facets oriented by the angle $a$. Thus, this function includes the topological description of the surface in a probability distribution function. In Equation (5), the distribution function, $D(a)$, is expressed with the distribution function of Beckmann [8], used also in the BRDF model of Cook-Torrance [9]. A Gaussian distribution of the slopes of the microfacets is considered. The slope is also the tangent of the angle $a$. The distribution is centred (average slope null) and the distribution depends only on the standard deviation of the slopes, noted $m$. This function is detailed in Equation (7).

$$
D\left(a^{r, x}, m\right)=\frac{\exp \left(-\frac{\tan ^{2} a^{r, x}}{2 m^{2}}\right)}{2 \pi m^{2} \cos ^{4} a^{r, x}}
$$


where $a^{\vec{r}, \vec{x}}$ is the facet angle (angle between $H$ and $N$ ) which reflect the light from the direction $L^{r}$ to the direction $L^{x}$. Exponents are ommited $(a)$ for the specific directions $L^{i}$ and $L^{r}$.

Finally, according to Equations (5), (7) and (6), the two bidirectional reflectivities $\rho_{\lambda}^{i, r}$ and $\rho_{\lambda}^{r, x}$ of the BRDF model become:

$$
\rho_{\lambda}^{i, r}=\frac{f_{\lambda}^{0} D(a, m)}{4 \cos \theta^{i} \cos \theta^{r}} \quad \text { and } \quad \rho_{\lambda}^{r, x}=\frac{F\left(\beta^{r, x}, n_{\lambda}, k_{\lambda}\right) D\left(\alpha^{r, x}, m\right)}{4 \cos \theta^{r} \cos \theta^{x}}
$$

\subsection{Writing of the diffusion function from the BRDF model}

The diffusion function of Equation (4) is rewritten according to the BRDF model of Equation (8) with the fixed angles $\left(\theta^{i}, \theta^{r}, \phi, a, \beta\right)$ for $\rho_{\lambda}^{i, r}$ (and, respectively, the angles $\left(\theta^{r}, \theta^{x}, \phi^{r, x}, a^{r, x}, \beta^{r, x}\right)$ for $\left.\rho_{\lambda}^{r, x}\right)$ :

$$
\eta_{\lambda}^{r, i}\left(m, n_{\lambda}, k_{\lambda}\right)=\frac{\cos \theta^{i}}{f_{\lambda}^{0} D(a, m)} \int_{2 \pi} F\left(\beta^{r, x}, n_{\lambda}, k_{\lambda}\right) D\left(\alpha^{r, x}, m\right) d \Omega^{x}
$$

For the experiment settings, $\theta^{r}=0^{\circ} ; a=\beta=\frac{\theta^{i}}{2} ; 0^{\circ} \leq a^{r, x}=\beta^{r, x}=\frac{\theta^{x}}{2} \leq 45^{\circ}$, the Fresnel function $F$ in the integral can also be approximated by the normal Fresnel factor $f_{\lambda}^{0}$. Indeed, with a detector aligned with the surface normal $\left(L^{r}=N\right.$ and $\left.\theta^{r}=0^{\circ}\right)$ and a light source close to the normal with an angle of $\theta^{i}=13^{\circ}$ the symmetry around $\mathrm{N}$ eliminates the need for the angle $\phi$. That case restricts the range of $\beta^{r, x}$ and thus only the micro-facets oriented with an angle below $45^{\circ}$ should contribute in the first reflection's BRDF. This means that a limit slope standard deviation is introduced, noted $m_{\text {lim }}$, to guarantee the energy conservation of the model which does not include other reflection orders. Also, with this criterion on $m$ and by using the normal Fresnel factor, the integral can be simplified and the diffusion function of Equation (9) becomes:

$$
\eta^{r, i}(m)=\frac{\cos \theta^{i}}{D(a, m)} \int_{2 \pi} D\left(a^{r, x}, m\right) d \Omega^{x}=8 \pi m^{2} \cos \theta^{i} \cos \theta^{r} \cos ^{4} a \exp \left(\frac{\tan ^{2} a}{2 m^{2}}\right)
$$

The following numerical application presents this calculation for platinum with the refractive indexes taken from [10] $\left(n_{\lambda}=5.5\right.$ and $k_{\lambda}=6.67$ at $\left.\lambda=2 \mu m\right)$ :

- $f_{\lambda}^{0} \approx F\left(13^{\circ}, n_{\lambda}, k_{\lambda}\right)=0.7461$ and $F\left(45^{\circ}, n_{\lambda}, k_{\lambda}\right)=0.7373$ (1.18\% relative error)

- $m_{\lim }=0.2$ with a relative error below $0.1 \%$ between $\eta^{r, i}(m)$ (Equation 10) and $\eta_{\lambda}^{r, i}\left(m, n_{\lambda}, k_{\lambda}\right)$ (Equation 9)

To summarise, starting in the framework of the geometrical optics approximation, a diffusion function based on an analytical BRDF model has been built. This model describes the roughness through a Gaussian model of the slope distribution. Without approximation, the diffusion function depends on one roughness parameter and the optical index of the surface. Furthermore, this expression can be simplified since the angular dependence of the Fresnel equation comes up for a large reflexion angle. Using the fact that the thermoreflectometer uses a small incident angle, we obtain the final expression of the diffusion function (Equation 10). 
In this approximation, the diffusion function no longer depends on the wavelength. This means that the physical origin of the wavelength dependence of the diffusion function is outside the framework of the model presented here. The only variable is the standard slope deviation $m$ which must be below a limit $\left(m_{\text {lim }}\right)$ associated with the setting. This limit, found in the numerical application, allows for a wide range of isotropic surface finishes. For very rough material with $m>m_{\text {lim }}$, the approximation of the Fresnel term and the neglecting of the multi-reflection are no more relevant and additional terms would be required in the diffusion function model.

In the next part, the diffusion function of Equation (10) is simulated as a function of the roughness parameter and for two geometrical setting.

\subsection{Simulation of the diffusion function versus the roughness parameter}

The objective is to study the shape of the diffusion function presented in Equation (10) and depending only on the standard deviation of the slopes $m$ and the angular setting. The two geometrical settings are presented below:

- Symmetrical setting noted $\eta^{13,13}$ with $\left(\theta^{i}, \theta^{r}, \phi\right)=\left(13^{\circ}, 13^{\circ}, 180^{\circ}\right)$

- Asymmetrical setting noted $\eta^{0,13}$ with $\left(\theta^{i}, \theta^{r}, \phi\right)=\left(13^{\circ}, 0^{\circ}, 180^{\circ}\right)$

The diffusion function models are plotted in Figure 3 with two near-angular settings to evaluate the possible dispersion of the experimental set-up.

For the symmetrical setting, the evolution of the diffusion function model is monotonic and increasing with $m$. The diffusion function then grows with the spreading of the slope distribution of the surface. The dispersion of the diffusion function value is low versus the angular dispersion of the geometrical setting. The diffusion function model is not greatly affected by the change of the angle as long as the symmetry is preserved. Thirdly, a single value of the roughness parameter $m$ can be estimated from experimental values of the

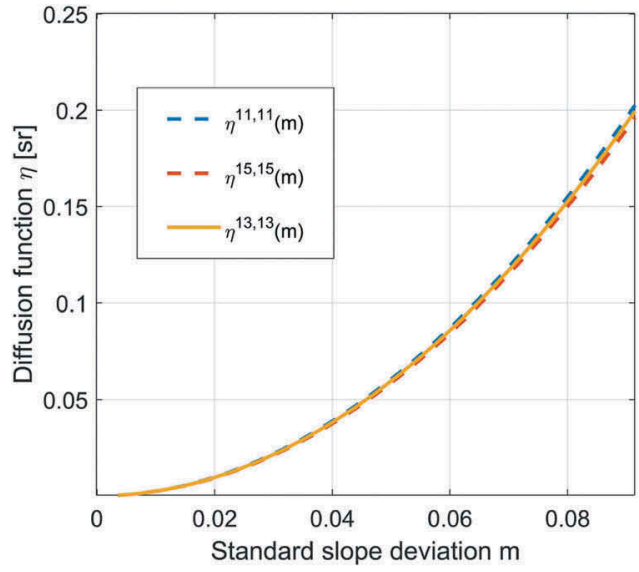

a

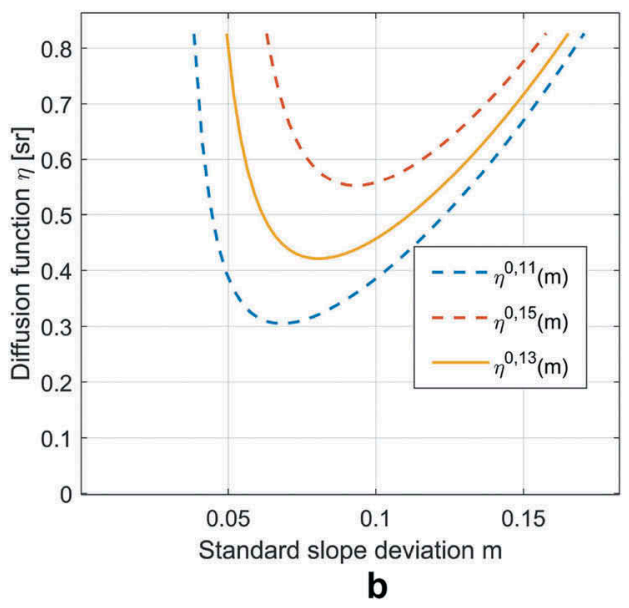

b

Figure 3. Diffusion function depending on the geometrical setting and the roughness parameter $m$. 
diffusion function. Since the geometrical setting is known, this estimation can be performed by bi-chromatic thermoreflectometry.

The asymmetric curves of the diffusion function model are presented in Figure 3(b) with, again, two near-angular settings. Firstly, the shape of the curves of the diffusion function is parabolic with a non-zero minimum. Secondly, the curve is very sensitive to the angle set, especially for a low value of $m$. A small variation in the angle causes a strong variation in the diffusion function. The correlation between the diffusion function and $m$ is thus not as simple as in the symmetrical setting. Two different values of $m$ can give the same value of the diffusion function and reciprocally. This is due to the possible intersection of two BRDF with different $m$ at asymmetric setting. Indeed, the higher the $m$ value is, the lower the maximum reflectivity is. However, as the scattering increase when $\mathrm{m}$ increase, this trend can be inverted for some asymmetric setting. Since the diffusion function is defined by the ratio of the reflectance to the bidirectional reflectivity (cf. Equation (4)) this possible BRDF intersection creates then two identical diffusion function values for two different $m$. This case can be observed in the asymmetric setting, but not in the symmetric setting because the maximum value of reflectivity is always selected.

Finally, two behaviours of the diffusion function were observed, depending on the choice of the angular setting. In the symmetrical case, a roughness parameter can be identified thanks to the monotonic growth of the diffusion function. This setting has also a low dependence on the angle. In the asymmetric case, the parabolic shape and its high sensitivity to the setting angle forbids all extrapolations of the standard slope deviations based on bi-chromatic thermoreflectometric measurement.

The next part of the article is devoted to the experimental validation of this modelling for a reference sample. It starts with a study of the roughness and the BRDF shape to finish with the application of bi-chromatic thermoreflectometry in the same geometrical setting as the presented simulations.

\section{Validation of the modelling for a reference sample}

In this section, experiments on a reference platinum sample are presented in order to validate the BRDF model of Equation (8), diffusion function of Equation (10) which is built from the BRDF model and the roughness parameter appearing in both functions. Firstly, the study of the roughness gives the experimental shape of the slope distribution and a reference value of the standard slope deviation value $m$. Then, the experimental BRDF measurement by a Fourier transform spectrometer validates the shape of the BRDF model. It also gives an estimation of the roughness parameter by a curve fitting. Finally, bi-chromatic thermoreflectometry is applied to the heated platinum sample. This opens a discussion on the roughness parameter values which appear in the roughness measurement, BRDF fitting and thermoreflectometry.

\subsection{Measurement of the platinum roughness}

The behaviour of the BRDF is illustrated on a pure platinum sample (pure at $99.95 \%$ ) with a controlled surface state. This metal was chosen because it does not oxidise and its optical properties are well documented in the literature. The sample was sandblasted to obtain a non-specular and isotropic optical behaviour. The measurement of the 
roughness was performed on a confocal profilometer operating with white light. 2D measurements confirmed the isotropy of the roughness and thus only a profile measurement is needed. A statistical study has been performed to determine the minimum length of the profile to obtain statistical representativeness. Varying the length of the profile, it has been shown that lengths superior to $2 \mathrm{~mm}$ show no bias. Thus, a length of $20 \mathrm{~mm}$ has been selected.

Figure 4 presents a part of the roughness profile of the sample with the detailed expression of the slope calculation, which is the growth rate on each point of the profile. The standard deviation $(m)$ associated with these slopes is also calculated and the formula is given in Figure 4. Figure 5 shows the experimental distribution of slope normalised as a density probability function (i.e. the sum of the bar's areas is one).

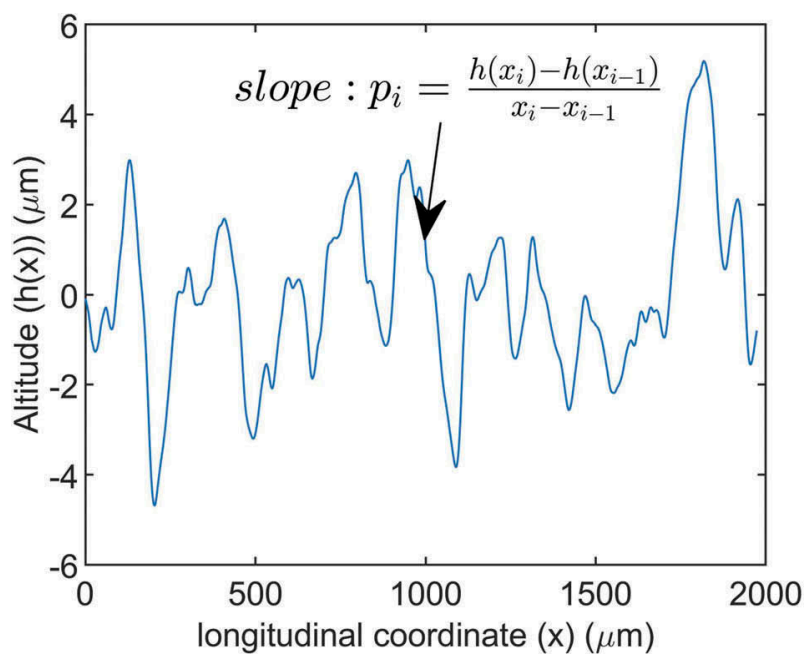

Figure 4. Roughness profile of the platinum sample.

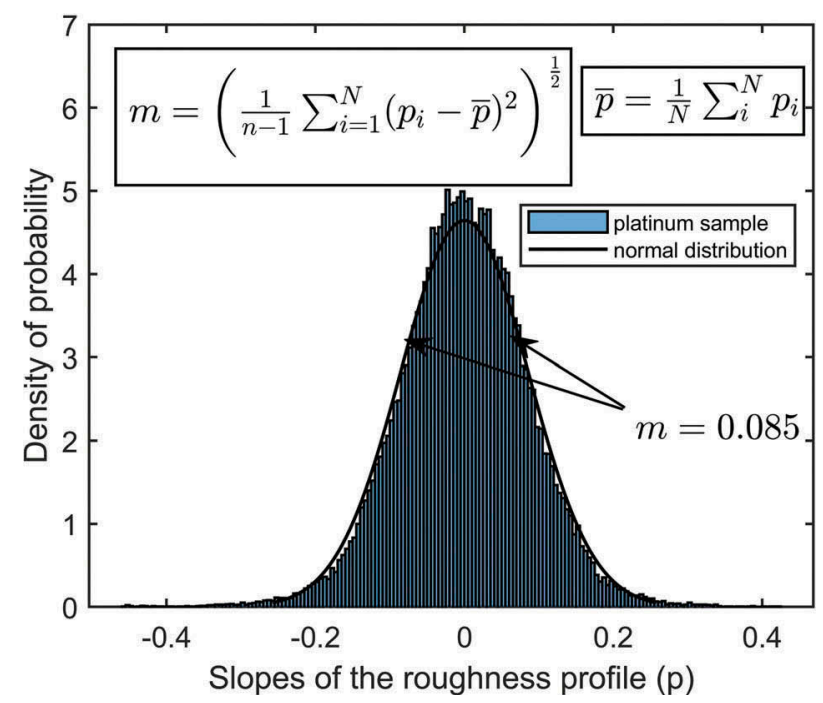

Figure 5. Density probability of the slope and Gaussian model. 
The experimental distribution of the slopes is centred and follows a Gaussian distribution, also plotted in Figure 5. The resulting standard slope deviation of $m=0.085$ is also below the defined limit of the model of Equation (10) $\left(m_{\text {lim }}=0.2\right)$ presented in subsection 3.3. The Beckmann's model chosen for modelling is therefore suitable for this sample.

The next part is devoted to a comparison between the BRDF model and measurement on this sample.

\subsection{Comparison of the BRDF model and measurement plan of incidence}

The BRDF of the platinum sample was measured using a Bruker ${ }^{\circledR}$ Fourier transform spectrometer (model Vertex 70) coupled to a goniometer module able to perform bidirectional reflectivity measurements in the plane of incidence. The signal was acquired (eight scans averaged) by a DLaTGS detector which is sensitive from 2 to $20 \mu \mathrm{m}$. The source is an halogen lamp. The angle of the incident flux $\left(\theta^{i}\right)$ is fixed at $13^{\circ}$ and the angle of the detector moves between $13^{\circ}$ and $89^{\circ}\left(\phi=180^{\circ}\right)$ with a step of $1^{\circ}$. The bidirectional reflectivity measurement of the platinum sample is achieved by comparison with a known bidirectional reflectivity of a reference sample, a diffusing gold coating. Experimental BRDF is presented in Figure 6 and compared with the BRDF model of Equation (8) fitted by a least square method. The values of the identified parameters are $m=0.0896$ and $f_{\lambda}^{0}=0.535$ at the wavelength $\lambda=2 \mu \mathrm{m}$.

The first observation is that the measured curve and the one estimated from the model are close for the angles below $35^{\circ}$. However, the error between the two curves is higher for the angle above $35^{\circ}$. The fact that multi-reflection phenomena in the BRDF model are neglected (see section 3) can partly explain this difference. Another cause may be the use of the Gaussian model of the slopes' probability density function which is not perfectly exact.

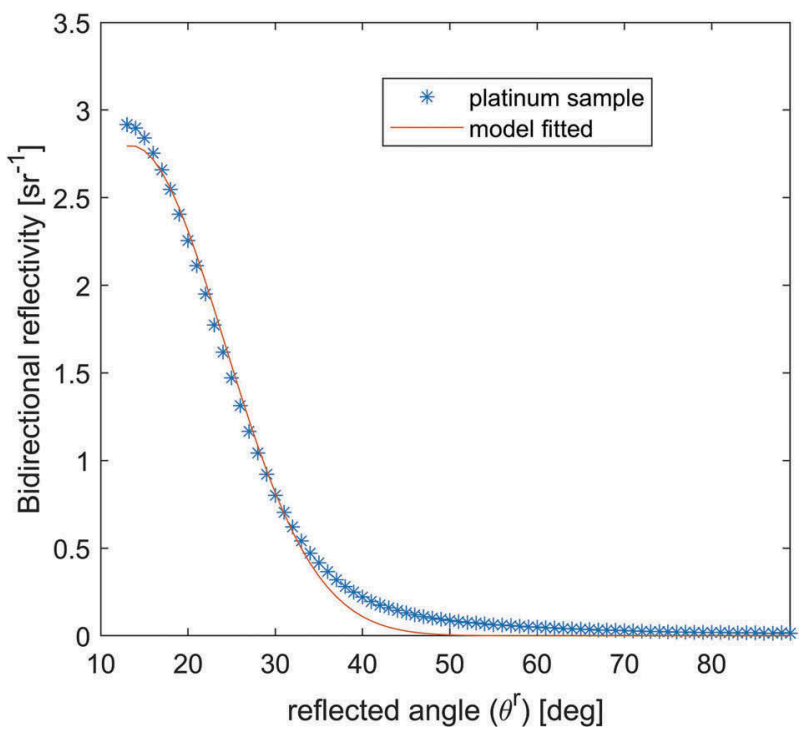

Figure 6. Measured BRDF of the platinum sample $\left(\theta^{i}=13^{\circ}\right)$ and BRDF model of Equation (8) fitted. 
These fitted values can also be compared to the measured value of the previous paragraph $m=0.085$ and to the literature value [10] $f_{\lambda}^{0}=0.746$. The relative error between the fitted and measured value of standard deviation, $m$, is $5.5 \%$ (and, respectively, between the fitted and reference value of the normal Fresnel factor, $f_{\lambda}^{0}$, is $28.3 \%$ ). The small relative error between the two slopes' standard deviation, $m$, means that the roughness parameter $m$ is well adapted to characterise the distribution of the BRDF of this sample. The biggest relative error on $f_{\lambda}^{0}$ may come from an error between the optical properties of our sample and those of the literature. It is also linked to the curve-fitting procedure which has led to an underestimation of $f_{\lambda}^{0}$ in order to have the minimum sum of squared residuals.

Finally, this comparison shows that the modelling approach for the BRDF is well adapted for this sample and especially for small angles, which correspond to our thermoreflectometry experimental setup. In the next subsection, thermoreflectometry will be applied with the model to link the experimental diffusion function to the roughness.

\subsection{Diffusion function estimated by bichromatic thermoreflectometry on the platinum sample}

The platinum sample studied previously was heated at $530^{\circ} \mathrm{C}$ with the experimental setup shown in Figure 7. The same geometrical settings as in the simulation presented in subsection 3.4 were used ( $\eta^{13,13}$ for symmetrical and $\eta^{0,13}$ for asymmetrical).

A bi-chromatic thermoreflectometry measurement was performed. The radiance temperature and bidirectional reflectivity images were captured by a Xenics Xeva NIR camera with an InGaAs matrix detector ( 320 x 256 pix) sensitive from 0.9 to $1.7 \mu \mathrm{m}$. The wavelength selection was made by three interferential filters. Each filter has a bandwidth of 50 $\mathrm{nm}$, respectively, centred at wavelengths 1064, 1310 and $1550 \mathrm{~nm}$. Three lasers with the same wavelength as the filters and with a bandwidth of $1 \mathrm{~nm}$ were coupled in optical fibre and connected to a projector. The size of the illuminated area was similar to the sample diameter $(30 \mathrm{~mm})$.

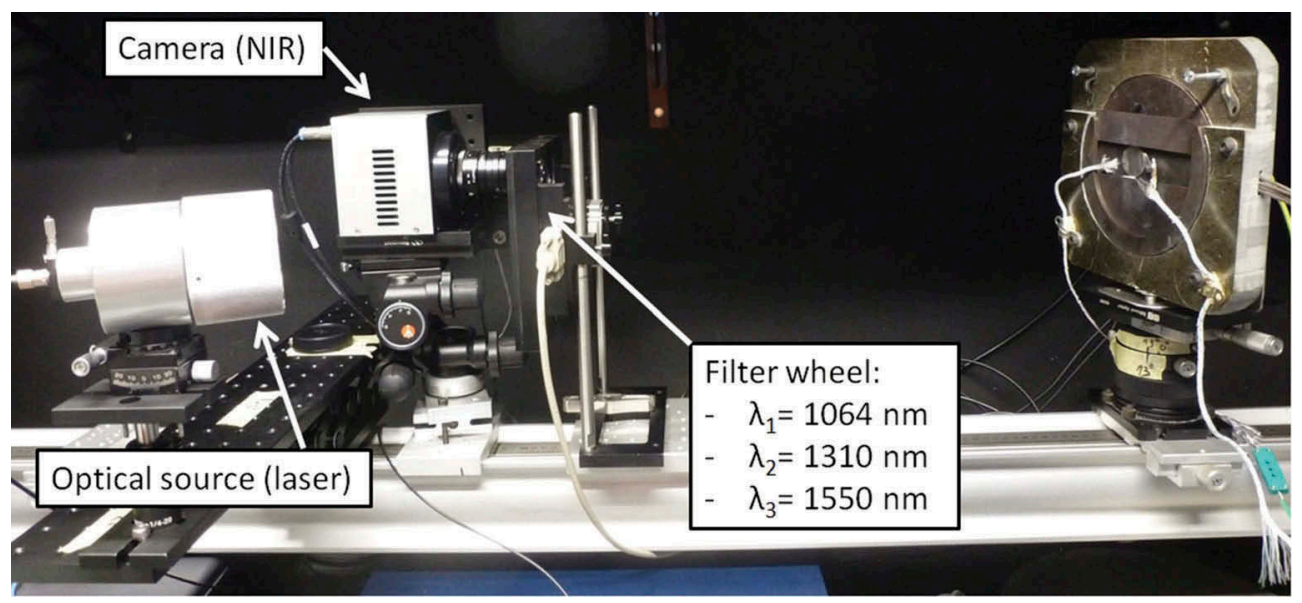

Figure 7. Experimental apparatus with $\theta^{i}=\theta^{r}=13^{\circ}$ and $\varphi=180^{\circ}$. 
For each setting, the radiance temperatures field and the bidirectional reflectivities field were measured at the three wavelengths of the filter wheel. A detailed description of the radiometric and reflectivity calibrations used can be found in [3]. Then, the thermoreflectometric system (Equation (3)) was solved on each pixel at the three wavelength couples with a constant diffusion function model. An area of 70 pixels (equivalent to a $2.5 \mathrm{~mm}$ side square) where the radiance temperatures and bidirectional reflectivities were homogeneous was defined. The average of all diffusion constants computed for each wavelength couple and settings in this area are presented for the temperature $530^{\circ} \mathrm{C}$ in Table 1. More details about the solving method can be found in [3].

As expected, because of its definition (see Equation 4), the diffusion function has a strong dependence on the geometrical setting. The diffusion function is nearly four times higher in the asymmetrical setting than in the symmetrical. Indeed, the higher values of bidirectional reflectivities are naturally measured with the symmetrical setting. As the sample was heated at the same temperature in both settings, all the changes in reflectivity are absorbed by the diffusion function in order to lead to a similar identified true temperature and associated emissivity. That is why the emissivities in Table 1 are consistent between the two settings.

Another remark is that the dispersion of the diffusion functions between all wavelength couples is low (2.5\% relative difference for $\eta^{13,13}$ and $4.5 \%$ for $\eta^{0,13}$ ). A decreasing of the diffusion function with the increasing of the wavelength couple is also observed in both settings. This could come from a slight wave-optics effect which can affect the shape of the BRDF.

Other measurement have been performed on the same sample at $500^{\circ} \mathrm{C}, 600^{\circ} \mathrm{C}$ and $700^{\circ} \mathrm{C}$. Referring to the measurement at $500^{\circ} \mathrm{C}$, the maximum relative variation of the identified the diffusion function was $-4.8 \%$. Since the surface state of the sample was constant during the experiment (same roughness and no oxidation), all the variations observed are due to the temperature dependency of the refractive index and/or the measurement error. Here, the maximum value is close to the variations observed for two wavelength couple at the same temperature (presented in Table 1). Thus, measurement error is the best explanation of the diffusion function variation. This also agree with the model which does not depend on the temperature.

As it was observed in the simulation of the diffusion function of Equation (10) (see 3.4), the symmetrical setting allows a direct estimation of the standard deviation slope $(m)$ by bi-chromatic thermoreflectometry, thanks to the monotonic growth of this function with regard to the roughness parameter. The resulting $m$ from thermoreflectometry are presented and compared with the two previous ones in the next subsection.

Table 1. Averaged diffusion function and directional emissivity identified by thermoreflectometry.

\begin{tabular}{|c|c|c|c|c|}
\hline $\begin{array}{l}\text { Wavelength } \\
\text { Couple [nm] }\end{array}$ & $\begin{array}{l}\text { Diffusion } \\
\text { Function } \\
\eta^{13,13}[\mathrm{sr}]\end{array}$ & $\begin{array}{l}\text { Diffusion } \\
\text { Function } \\
\eta^{0,13}[\mathrm{sr}]\end{array}$ & $\begin{array}{c}\text { Directional } \\
\text { Emissivity } \varepsilon^{13}\end{array}$ & $\begin{array}{l}\text { Directional } \\
\text { Emissivity } \varepsilon^{0}\end{array}$ \\
\hline$\lambda_{1} \lambda_{2}(1064,1310)$ & 0.1386 & 0.5245 & $0.36,0.32$ & $0.36,0.32$ \\
\hline$\lambda_{1} \lambda_{3}(1064,1550)$ & 0.1362 & 0.5073 & $0.37,0.32$ & $0.38,0.33$ \\
\hline$\lambda_{2} \lambda_{3}(1310,1550)$ & 0.1352 & 0.5007 & $0.33,0.33$ & $0.34,0.33$ \\
\hline
\end{tabular}


Table 2. Comparison of the roughness parameter estimated by thermoreflectometry, curve fitting and roughness measurement.

\begin{tabular}{lccc}
\hline Method & $m=f\left(\eta_{\lambda_{1}: \lambda 2}^{13,13}\right)$ & $m=f\left(\eta_{\lambda_{1} ; \lambda 3}^{13,13}\right)$ & $m=f\left(\eta_{\lambda_{2} ; \lambda 3}^{13,13}\right)$ \\
\hline Thermoreflectometry & 0.0753 & 0.0755 & 0.0762 \\
Curve fitting & & 0.0896 at $\lambda=2 \mu \mathrm{m}$ & \\
Roughness measurement & & 0.085 at $\lambda=2 \mu \mathrm{m}$ & \\
\hline
\end{tabular}

\subsection{Discussion on the roughness parameters}

Results of the identification of the roughness parameter by thermoreflectometry, in the symmetrical setting, are shown in Table 2 with the measurement on a roughness profile in subsection 4.1 and the identification made by curve fitting on measured BRDF in subsection 4.2.

As in the result in Table 1, the $m$ identified by thermoreflectometry have a low dispersion since they are between 0.0753 and 0.0762 . These values are quite consistent compared to the measured one on a roughness profile (subsection $4.1 \mathrm{~m}=0.085$ ) and the estimated one by curve fitting (subsection $4.2 m=0.0896$ ). However, the identified roughness parameters by thermoreflectometry are approximately $10 \%$ below the value measured on the roughness profile. This could mostly be explained by the misalignment of the sample during the thermoreflectometry measurement, which could affect the diffusion function and thus the extrapolated roughness. It may also be caused by nonhomogeneities from the spot and the surface illuminated in the bidirectional reflectivity measurement step. Despite this, the information on the roughness brought by the model seems promising for an application of "in-situ" monitoring of the surface state during thermo-mechanical stress, for example.

\section{Conclusion}

In the framework of the development of thermoreflectometry, the choice of the diffusion function with regard to the materials studied is essential for the accuracy and validity of the method. In this article, a diffusion function, based on a physical model of BRDF, which includes a roughness parameter and the optical property of the material, was detailed. The BRDF model describes the case of rough metallic surfaces with a roughness size compatible with the geometrical optics approximation. Then, with the appropriate angular setting and roughness, it can be shown that this diffusion function model does not depend on the wavelength but only on the slope distribution of the surface facets. The simulation of this model in a symmetrical setting shows that it is possible to estimate the roughness parameter from bi-chromatic thermoreflectometry.

Validations of the roughness parameter used in the BRDF and diffusion function model were carried out on a reference platinum sample. Firstly, the reference roughness parameter was calculated from a measured roughness profile. Then, the BRDF model used to build the diffusion function was fitted on an experimental BRDF. It validated its shape and also the roughness parameter. The identified value was close to the reference value. Lastly, bi-chromatic thermoreflectometry was applied on the heated platinum sample in 
a symmetrical setting. The use of the new diffusion function then provided a roughness parameter consistent with the two previous values.

These results will have to be validated on other samples. Also, some improvements on the model need to be included to take into account the spectral dependence of the diffusion function. Finally, the new information brought by the model will be exploited in order to follow and interpret the surface state change on aeronautic alloy during thermomechanical stress.

\section{Disclosure statement}

No potential conflict of interest was reported by the authors.

\section{Notes on contributors}

Benjamin Javaudin, born in 1992, studied thermal and energy sciences at the graduate school of engineering of the University of Nantes and graduated in 2016. Subsequently, he started his PHD in the research group Metrology Identification Control and Monitoring of the Clement Ader Institute at IMT Mines Albi where he worked on the development of the thermoreflectometry method.

Rémi Gilblas defended his PhD in 2012 about the development of a new non contact measurement method called thermoreflectometry. After a post-doctoral research about the detection of microcracks on highly specular components used for spatial applications, he occupies a research engineer position in the Clement Ader Institute, a french research laboratory focused on aeronautical structures and materials. His skills are mainly linked to radiative properties of materials at high temperature, thermal metrology and instrumentation.

Thierry Sentenac is assistant professor at IMT Mines Albi since 1995 and he carries out his research work in the Clement Ader Institute (ICA-CNRS 5312) laboratory (250 people) and in the Laboratory for Analysis and Architecture of Systems (LAAS-CNRS) (600 people). From 2002 to 2008, he was head of the Electrical Engineering and Automatic Control department in IMT Mines Albi. From 2010 to 2019, He was head of program master in Advanced Materials and Mechanical Engineering. He is head of Innovative Optical Methods for Dimensional and Thermal Metrology research group (15 people) dedicated to the development of non-contact methods for mechanical and thermal measurements in the field of material processing, for the study of the behaviour of materials and structures, and for process monitoring. He is also the Deputy Director of the ICA Lab and the head the ICA-Albi Research Center (70 people). Working in the Robotics group of LAAS-CNRS (Toulouse, France) on the application of computer vision and infrared cameras to the monitoring of aircraft cargo holds, he received his PhD in Computer Vision in 2002 from Université de Toulouse (Toulouse, France). Accreditation to supervise research of the Université de Toulouse since 2014, Thierry Sentenac was the director or co-director of fifteen $\mathrm{PhD}$ students in the field of full-field thermal measurements, 3D reconstruction, control, monitoring and visual servoing. His main interest topics are computer vision, robotic, full-field thermal measurements and smart material.

Yannick Le Maoult, after five years as a research engineer in the industry, Y. Le Maoult has defended his PHD in 1992, PHD involving infrared thermography applied to combustion gases. Since 1995, Dr. Le Maoult has worked as an associate professor at IMT-Mines Albi and carried out his research work at theClement Ader Institute Albi (ICA-Albi). He was appointed full professor in 2012 in the same institute. Professor Le Maoult is now the head of the MICS Research group as well as the head of MiMauSa research and engineering platform (ICA-Albi). Professor Le Maoult is involved in infrared radiation physics and more generally heat transfer problems, optical properties of solids and infrared radiometry. 


\section{References}

[1] Herve P, Veillard L, Morel A. Radiométrie: L'ultraviolet (radiometry the ultraviolet). Revu pratique de contrôle industriel. 1997;36(205):60-90.

[2] Duvaut T. Comparison between multiwavelength infrared and visible pyrometry: application to metals. Infrared Phys Technol. 2008;51:292-299.

[3] Sentenac T, Gilblas R, Hernandez D, et al. Bi-color near infrared thermoreflectometry: a method for true temperature field measurement. Rev Sci Instrum. 2012;83(12):124902.

[4] Gilblas R, Sentenac T, Hernandez D, Le Maoult Y. Quantitative temperature field measurements on a non-gray multi-materials scene by thermoreflectometry. Infrared Phys Technol. 2014;66:70-77.

[5] Tang K, Dimenna RA, Buckius RO. Regions of validity of the geometric optics approximation for angular scattering from very rough surfaces. Int J Heat Mass Transfer. 1996;40:49-59.

[6] Torrance KE, Sparrow EM. Theory for off-specular reflection from roughened surfaces. JOSA. 1967;57(9):1105-1114.

[7] Bergstrom D, Powell J, Kaplan AFH. A ray-tracing analysis of the absorption of light by smooth and rough metal surfaces. J Appl Phys. 2007;101(11):113504.

[8] Beckmann P, Spizzichino A. The scattering of electromagnetic waves from rough surfaces. New York: Macmillan; 1963.

[9] Cook RL, Torrance KE. A reflectance model for computer graphics. ACM SIGGRAPH Comput Graphics. 1981;15:307-316.

[10] Rakić $A D$, Djurišic $A B$, Elazar JM, et al. Optical properties of metallic films for vertical-cavity optoelectronic devices. Appl Opt. 1998;37:5271-5283. 